\title{
Propuesta sobre la Inclusión y Eliminación de Términos Anatómicos en Terminologia Anatomica Correspondientes a la Articulación Temporomandibular
}

\author{
Proposal on Inclusion and Elimination of Anatomical Terms in \\ Terminologia Anatomica corresponding to the Temporomandibular Joint
}

Ramón Fuentes ${ }^{1}$ \& Nicolás Ernesto Ottone ${ }^{1,2}$

FUENTES, R. \& OTTONE, N. E. Propuesta sobre la inclusión y eliminación de términos anatómicos en Terminologia Anatomica correspondientes a la articulación temporomandibular. Int. J. Morphol., 35(1):12-15, 2017.

RESUMEN: En esta comunicación breve se plantea como objetivo la necesidad de analizar los términos anatómicos relacionados a la articulación temporomandibular presentes en Terminologia Anatomica Internacional (TAI) y, en referencia a estos, incluir nuevos términos no presentes en la TAI, eliminar términos no correctos de la TAI y analizar otros términos que son identificados en la literatura científica pero que no están incluidos en Terminologia Anatomica.

PALABRAS CLAVE: Terminologia Anatomica; Articulación temporomandibular.

\section{INTRODUCCIÓN}

Desde su origen, Terminologia Anatomica ha sido sujeto de conflictos y desacuerdos. Durante siglos los anatomistas trabajaron aislados, denominando a las distintas estructuras por analogía de formas, con sus propios nombres o los de sus maestros. Algunas estructuras llegaron a tener varias denominaciones y la nomenclatura anatómica se colmó de nombres, sinónimos y epónimos. A fines del siglo XIX, existían diez términos para cada estructura, creándose de esta manera gran confusión y caos en la terminología de ese tiempo, lo que no permitía un fluido intercambio de información y comunicación. Es así que en 1895, en Basilea, se creó el primer listado de términos anatómicos, denominado Nomina Anatomica, Basilea Nomina Anatomica (BNA) en inglés o Nomenclatura Anatomica Internacional de Basilea en español. A partir de este hito, comenzó a trabajarse en el perfeccionamiento de la terminologia hasta que en 1989, la Federación Internacional de Asociaciones de Anatomía desarrolla Terminologia Anatomica Internacional (TAI), que se encuentra redactada en latín y a partir de la cual se debe realizar la traducción al idioma correspondiente. Además establece que: los nombres de las estructuras deben tener un valor informativo; eliminación de epónimos y homónimos para evitar confusiones; las estructuras en las mismas regiones anatómicas de- ben tener nombres armonizados (FICAT, 1998; FCAT, 2001; Vásquez \& del Sol, 2015).

De esta manera, y tomando en cuenta la importancia de mantener un idioma común anatómico, consideramos fundamental su establecimiento en una región anatómica de extrema importancia, como es la articulación temporomandibular (ATM). El objetivo de esta breve comunicación fue analizar la TAI en relación a los términos asociados a la ATM, presentes en la misma, y la identificación de términos ausentes, que deberían ser incorporados, como así también términos que deberían eliminarse o no considerarse como parte de la ATM.

\section{RESULTADOS Y DISCUSIÓN}

En la Tabla I se pueden observar todos los términos anatómicos asociados a la ATM presentes en la TAI. El término cápsula articular se encuentra incluído en la sección Juncturae; Systema articulare / Articulaciones; Sistema articular (A03.0.00.000). Sería adecuado en relación a este término su inclusión en la misma sección que articulación

\footnotetext{
${ }^{1}$ CICO - Centro de Investigación en Ciencias Odontológicas, Facultad de Odontología, Universidad de La Frontera, Temuco, Chile.

${ }^{2}$ Programa de Doctorado en Ciencias Morfológicas, Facultad de Medicina, Universidad de La Frontera, Temuco, Chile.
} 
Tabla I. Términos anatómicos presentes en TAI relacionados y correspondientes a la articulación temporomandibular.

\begin{tabular}{lll}
\hline Código TAI & \multicolumn{1}{c}{ Latín } & \multicolumn{1}{c}{ Español } \\
\hline A03.0.00.000 & Juncturae; Systema articulare & Articulaciones; Sistema articular \\
A03.0.00.001 & Junctura & Articulación \\
A03.0.00.020 & Junctura synovialis; Articulatio; Diarthrosis & Articulacio_n sinovial; Diartrosis \\
A03.0.00.022 & Cavitas articularis & Cavidad articular \\
A03.0.00.023 & Fossa articularis & Fosa articular \\
A03.0.00.026 & Capsula articularis & Cápsula articular \\
A03.0.00.028 & Membrana synovialis; Stratum synoviale & Membrana sinovial; Capa sinovial \\
A03.0.00.032 & Discus articularis & Disco articular \\
A03.0.00.034 & Ligamenta & Ligamentos \\
A03.0.00.035 & Ligg. intracapsularia & Ligs. intracapsulares \\
A03.0.00.037 & Ligg. extracapsularia & Ligs. extracapsulares \\
A03.1.06.001 & Articulationes cranii & Articulaciones sinoviales del cra_neo \\
A03.1.07.001 & Articulatio temporomandibularis & Articulacio_n temporomandibular \\
A03.1.07.002 & Discus articularis & Disco articular \\
A03.1.07.003 & Lig. laterale & Lig. lateral \\
A03.1.07.004 & Lig. mediale & Lig. medial* \\
A03.1.07.005 & Membrana synovialis superior & Membrana sinovial superior \\
A03.1.07.006 & Membrana synovialis inferior & Membrana sinovial inferior \\
A03.1.07.007 & Lig. sphenomandibulare & Lig. esfenomandibular \\
A03.1.07.008 & Lig. stylomandibulare & Lig. estilomandibular \\
A05.3.01.102 & Raphe pterygomandibularis & Rafe pterigomandibular** \\
& &
\end{tabular}

* Lig. medial: debería eliminarse de la TAI, en relación a la ATM. ** No forma parte de la ATM, correctamente incluído en FARINGE (A05.3.01.001).

temporomandibular (A03.1.07.001), pero dependiendo de éste término, más allá de su presencia en la sección general de sistema articular. Por su parte, el término articulación temporomandibular se encuentra incluído en la sección Articulationes cranii / Articulaciones sinoviales del cráneo (A03.1.06.001). Debajo del mismo son incluídos los siguientes términos: Disco articular (A03.1.07.002), Lig. lateral (A03.1.07.003), Lig. medial (A03.1.07.004), Membrana sinovial superior (A03.1.07.005), Membrana sinovial inferior (A03.1.07.006), Lig. esfenomandibular (A03.1.07.007), Lig. estilomandibular (A03.1.07.008). En relación a estos términos, los mismos si bien están bien incluídos por debajo del término articulación temporomandibular, deberían incluirse como dependencia del mismo, algo que no sucede en la terminología sugerida por la Sociedad Anatómica de España (2001). En la Tabla II se identifican las estructuras anatómicas que son nombradas en la literatura como pre- sentes en la ATM pero que no están incluidas en la TAI. En referencia a estos términos no considerados en la TAI, se encuentran los siguientes: ligamento posterior, ligamentos colaterales, ligamento de Tanaka y ligamento de Pinto.

Con respecto al ligamento posterior, es también conocido como "zona bilaminar" (Parsons \& Boucher, 1966; Hall et al., 1984; van der Kuijl et al., 1990; Hollender et al., 1998; Benigno et al., 2001; Zhang et al., 2006; Leonardi et al., 2012). Estos autores lo ubican en el área retrodiscal, permitiendo la unión entre la cápsula articular y el disco articular. Sin embargo, Mackinnon \& Morris (1993) y Pertes \& Gross (2005), lo nombran como ligamento posterior, e indican que limita los movimientos de protrusión de la mandíbula. Todos consideran la conformación de este ligamento posterior por dos tipos de fibras, elásticas y colágenas. De esta manera, consideramos adecuada la inclusión en la

Tabla II. Estructuras anatómicas asociadas a la ATM en la literatura, pero que no se encuentran incluídas en la TAI.

\begin{tabular}{ll}
\hline Estructura anatómica no presente en la TAI & Propuesta terminológica \\
\hline Ligamento posterior & Lig. posterior \\
Ligamentos colaterales & Lig. colateral lateral y Lig. colateral medial \\
Ligamento de Tanaka & (confirmar su existencia) \\
Ligamento de Pinto & (confirmar su existencia) \\
\hline
\end{tabular}


TAI del término ligamento posterior debido a la confirmación de su existencia morfológica.

Con respecto a los ligamentos colaterales, de acuerdo a diversos autores, pueden ser considerados verdaderos ligamentos de la articulación temporomandibular (Okeson, 2003; Fuentes et al., 2016). Están conformados por fibras de tejido conjuntivo colágeno, y se identifican uno lateral y otro medial, extendiéndose desde los respectivos márgenes del disco articular hasta el cóndilo de la mandíbula (Langton \& Eggleton, 1992; Okeson). Se diferencian morfológicamente entre sí, siendo el ligamento colateral lateral más delgado y amplio, con respecto a su homólogo medial (Langton \& Eggleton). Debido a esto, consideramos adecuada la inclusión en la TAI de estos ligamentos, como "Lig. colateral lateral" y "Lig. colateral medial".

En la literatura también suelen nombrarse dos términos anatómicos con epónimos. Por un lado, el ligamento de Pinto, descripto en el año 1962 como un pequeño ligamento que une el cuello y proceso anterior del martillo a la porción posterosuperior de la cápsula articular, disco articular y ligamento esfenomandibular (Fuentes et al., 2014a). Por otro lado se encuentra el ligamento de Tanaka, ubicado anteromedialmente, y extendiéndose desde el disco articular a la fosa mandibular, fue definido así por Langton \& Eggleton, originalmente descubierto por Terry Tanaka, a comienzos de los años 80 del siglo XX. Sin embargo, estos dos ligamentos aún no cuentan con suficiente respaldo morfológico para considerar su presencia como normal, con una alta frecuencia de aparición o como una variación anatómica; por lo cual aún son considerados como hallazgos aislados en la disección anatómica. En esta misma situación se encuentra el ligamento medial (A03.1.07.004), el cual si bien está incluido en la TAI, el mismo no cuenta con suficiente evidencia morfológica como para asegurar su existencia (Fuentes et al., 2016). Por lo cual, debería eliminarse de la TAI.

En relación al rafe pterigomandibular (A05.3.01.102), éste se encuentra correctamente incluído en la sección Faringe (A05.3.01.001), por lo cual no debe ser considerado como una estructura anatómica perteneciente a la ATM (Fuentes et al., 2014b).

A su vez, en la sección de "Articulaciones; Sistema articular" (A03.0.00.000), se encuentran incluídos los términos anatómicos Ligs. intracapsulares (A03.0.00.035) y Ligs. extracapsulares (A03.0.00.037). Estos podrían incorporarse como dependiendo de "Articulación temporomandibular" (A03.1.07.001). A su vez, se podrían dividir los ligamentos de la ATM como intracapsulares y extracapsulares, dependiente de estos términos en el TAI. Por lo tanto, como dependencia del término "Ligs. intracapsulares" se podrían incluir los términos Lig. posterior, Lig. colateral lateral y Lig. colateral medial. Mientras, que como dependencia del término "Ligs. extracapsulares" se podrían incluir los términos Lig. esfenomandibular y Lig. estilomandibular. Y sería necesario analizar, si el ligamento lateral (descrito generalmente como ligamento temporomandibular) es, de acuerdo a su disposición, un ligamento capsular o extracapsular.

En conclusión, los puntos fundamentales de nuestra propuesta son los siguientes:

- Ligamento posterior: incorporación en la TAI como "Lig. posterior".

Ligamentos colaterales: incorporación en la TAI como "Lig. colateral lateral" y "Lig. colateral medial".

- Lig. medial (A03.1.07.004): eliminación de la TAI, no hay suficiente evidencia teórica, morfológica, ni experimental que avalen su presencia.

- Ligamentos de Tanaka y Pinto: no incluir en la TAI, porque requieren mayor estudio en cadáveres para su determinación.

- Ligs. Intracapsulares: incluirlo como dependiente del término Articulación temporomandibular (A03.1.07.001). Y dependiendo de este, los términos Lig. posterior, Lig. colateral lateral y Lig. colateral medial.

- Ligs. Extracapsulares: incluirlo dependiendo del término Articulación temporomandibular (A03.1.07.001). Y dependiendo de este, los términos Lig. esfenomandibular y Lig. estilomandibular.

- Lig. lateral: analizar si, de acuerdo a su morfología, es un ligamento capsular o extracapsular.

FUENTES, R. \& OTTONE, N. E. Proposal on inclusion and elimination of anatomical terms in Terminologia Anatomica corresponding to the temporomandibular joint. Int. J. Morphol., 35(1):12-15, 2017.

SUMMARY: The objective presented in this short communication is the need to analyze anatomical terms related to the temporomandibular joint as noted in the International Anatomical Terminology (IAT) and referring to these, include new terms not present in the IAT, removing the incorrect terms of the IAT and analyzing other terms that are identified in the scientific literature but are not included in Terminologia Anatomica.

KEY WORDS: Terminologia Anatomica; Temporomandibular joint. 
FUENTES, R. \& OTTONE, N. E. Propuesta sobre la inclusión y eliminación de términos anatómicos en Terminologia Anatomica correspondientes a la articulación temporomandibular. Int. J. Morphol., 35(1):12-15, 2017.

\section{REFERENCIAS BIBLIOGRÁFICAS}

Benigno, M. I.; Azeredo, R. A.; Lemos, J. L.; König Júnior, B. \& Liberti, E. A. The structure of the bilaminar zone in the human temporomandibular joint: a light and scanning electron microscopy study in young and elderly subjects. J. Oral Rehabil., 28(2):113-9, 2001.

Comité Federal sobre Terminología Anatómica (FCAT). Terminologia Anatomica. Terminología Anatómica Internacional. Madrid, Editorial Médica Panamericana, 2001.

Federative International Committee on Anatomical Terminologies (FICAT). Terminologia Anatomica. International Anatomical Terminology. New York, Thieme, 1998.

Fuentes, R.; Ottone, N. E.; Cantin, M. \& Bucchi, C. Análisis de los Términos Utilizados en la Literatura Científica para Referirse a los Ligamentos Extracapsulares de la Articulación Témporomandibular: Parte II: Ligamentum stylomandibulare y Raphe pterymandibulare. Int. J. Morphol., 32(4):1289-95, 2014a.

Fuentes, R.; Cantin, M. \& Bucchi, C. A study of terms used in scientific literature to refer to the extracapsular ligaments of the temporomandibular joint. Part I: Ligamentum sphenomandibulare. Int. J. Morphol., 32(3):981-6, 2014b.

Fuentes, R.; Ottone, N. E.; Bucchi, C. \& Cantin, M. Análisis de los términos utilizados en la literatura científica para referirse a la cápsula articular y ligamentos articulares de la articulación temporomandibular. Int. J. Morphol., 34(1):342-50, 2016.

Hall, M. B.; Brown, R. W. \& Baughman, R. A. Histologic appearance of the bilaminar zone in internal derangement of the temporomandibular joint. Oral Surg. Oral Med. Oral Pathol., 58(4):375-81, 1984.

Hollender, L.; Barclay, P.; Maravilla, K. \& Terry, V. The depiction of the bilaminar zone of the temporomandibular joint by magnetic resonance imaging. Dentomaxillofac. Radiol., 27(1):45-7, 1998.

Langton, D. P. \& Eggleton, T. M. Functional anatomy of the temporomandibular joint complex. Santiago de Chile, Inforc Publications, 1992.

Leonardi, R.; Rusu, M. C.; Loreto, F.; Loreto, C. \& Musumeci, G. Immunolocalization andexpression of lubricin in the bilaminar zone of the human temporomandibular joint disc. Acta Histochem., 114(1):15, 2012.

Mackinnon, P. C. B. \& Morris, J. F. Oxford Anatomía Funcional. Cabeza y Cuello. Volumen III. Bogotá, Editorial Médica Panamericana, 1993.

Okeson, J. P. Tratamiento de oclusión y afecciones temporomandibulares. $5^{\text {a }}$ ed. Madrid, Elsevier Mosby, 2003.

Parsons, M. T. \& Boucher, L. J. The bilaminar zone of the meniscus. $J$. Dent. Res.,45(1):59-61, 1966.

Pertes, R. \& Gross, S. Tratamento Clínico das disfunçoes Temporomandibulares e da dor Orofacial. São Paulo, Quintessence, 2005.

van der Kuijl, B.; Vencken, L. M.; de Bont, L. G. \& Boering, G. Temporomandibular joint computed tomography: development of a direct sagittal technique. J. Prosthet. Dent., 64(6):709-15, 1990.

Vásquez, B. \& del Sol, M. Terminologia Anatomica and Terminologia Histologica. A meeting point between morphologists. Int. J. Morphol., 34(4):1585-90, 2015.

Zhang, Y. K.; Gu, Z. Y. \& Hu, Q. G. Experimental study of the mechanism of chondroid metaplasia of the bilaminar zone following anterior disc displacement of temporomandibular joint. Shanghai Kou Qiang Yi Xue, 15(1):48-51, 2006.

\author{
Dirección para Correspondencia : \\ Dr. Ramón Fuentes \\ CICO - Centro de Investigación en Ciencias Odontológicas \\ Facultad de Odontología \\ Universidad de La Frontera \\ Manuel Montt 112, Casilla 54-D \\ Temuco \\ CHILE
}

Email: ramon.fuentes@ufrontera.cl

Recibido: 28-10-2016

Aceptado:24-11-2016 